\title{
Assessment of the Gliding of Extensor Tendons in Post-Traumatic Hand Defects Reconstructed Using Muscle Flaps Versus Fascio-Cutaneous Flaps
}

\author{
KHALED A. REYAD, M.D., M.R.C.S.*; HANY SAAD SETTA, M.D.* and HALA M. ABD EL-SABOUR, M.D.** \\ The Departments of Plastic, Reconstructive \& Maxillofacial Surgery* and Physical Medicine, Rheumatology \& Rehabilitation**,
} Faculty of Medicine, Ain Shams University

\begin{abstract}
Background: Use of fascio-cutaneous or fascial flaps are well known methods of reconstruction of dorsal hand defects to preserve the gliding movement of the extensor tendons. Whilst reconstruction of large hand defects extending to the wrist or the distal forearm entails use of larger flaps with large donor sites, so muscle flaps may have an advantage over the fascial or fascio-cutaneous flaps in coverage of these large defects. The aim of this study is to compare the gliding of the extensor tendons underneath fascio-cutaneous and muscle flaps on the dorsum of the hand.
\end{abstract}

Patients and Methods: The study included 20 patients with dorsal hand trauma with exposed extensor tendons, ten defects were reconstructed by free muscles flap and split thickness skin graft and the other ten were reconstructed with fascio-cutaneous flaps. Early rehabilitation in all cases was done by a single dedicated physiotherapist. Tendon gliding was assessed by measurement of active flexion and extension at the metacarpo-phalangeal joint using goniometer after complete flap healing four months following flap inset.

Results: There was no statistically significant difference in gliding for extensor tendons in patients with hand defects reconstructed by free muscle flaps and skin graft versus fasciocutaneous flaps.

Conclusion: Both fascio-cutaneous and free muscle flaps had comparable results concerning the extensor tendons gliding underneath.

Key Words: Hand trauma - Fasciocutaneous flaps - Muscle flaps - Extensor tendon gliding

\section{INTRODUCTION}

Fascial flaps are considered the best option for coverage of dorsal hand defects as it creates a gliding surface to facilitate tendon and joint mobility [1]. Multiple fascial flaps were used as lateral arm flap [2], radial forearm flap [3], temporo-parietal fascia [4], dorsal ulnar forearm[5], posterior interosseous flap [6], dorsal thoracic [7]. The use of muscle free flaps is well known especially for large defects as latissimus and rectus free flaps [8-11].

The gliding surface of the dorsum of the hand is crucial for tendon movement, but when there are large defects, options may be limited due to paucity of the donor tissues to supply flaps with huge dimensions especially in those defects involving the hand dorsum with wrist and distal forearm skin loss. So the use of large, robust muscle flaps to cover those large skin defects were introduced as free latissimus and rectus muscles.

Many studies had recorded the use of muscle, fascio-cutaneous, fascial and venous flaps and they found that fascia and muscle flaps scored equally in terms of overall aesthetics, color, and contour match, while fascio-cutaneous flaps had significantly worse aesthetic, contour, and color match results compared with all other flap types [12]. To our knowledge there is no any other study that compared in between these flaps in terms of tendon gliding. The tendons of the hand pose a particular challenge for the hand therapist, as full hand function requires the tendon to glide long distances [13].

\section{PATIENTS AND METHODS}

This prospective comparative study was done through September 2014 till January 2017. Written informed consent was obtained from each patient. All patients included were suffering from posttraumatic skin and soft tissues defect over the dorsum of the hand; with or without extension to the distal one third of the forearm; exposing the underlying tendons of all ages, no specified gender.

The exclusion criteria were any associated tendon injury or associated fractures, history of any related hand injuries or surgeries, history of rheumatoid arthritis, diabetes mellitus, or osteoporosis, the presence of central or peripheral nerve injuries.

The experimental methods were carried out in accordance with approved guidelines and regulations of our institution. 
Twenty patients shared in this study and they were divided into two groups the first group of patients' $(n=10)$ hand defects were early reconstructed by muscle flaps and split thickness skin grafts, the second group of patients' $(n=10)$ defects were early reconstructed using fascio-cutaneous flaps.

Early postoperative rehabilitation was the protocol following immobilization in the first 3-5 days after flap inset [14]. Passive range of motion guided by pain was applied, later on; at two weeks later scar stretching was done [16]. Once healed, active range of motion exercises coupled with ultrasound [17] and later on return to work [18].

The metacarpo-phalangeal joint active and passive range of motion angles were measured using goniometer after flap inset by four month in both groups. Statistical Analysis of the results using SPSS (statistical program for social science) as follows; Description of quantitative variables as mean and standard deviation (SD). Pearson's Correlation coefficient (Pearson's $r$ ) was used to rank different variables versus each other (where 1 or -1 are highly related with linear relationship, whilst 0 signifies no linear correlation). The $p$ value was calculated and correlated to the variants and $p$-value was considered insignificant if $>0.05$, significant if $<0.05$ and highly significant if $<0.01$.

\section{RESULTS}

The mean age for patients was $38.3 \pm 13.3$ years. Eighteen male and two female patients who were suffering from dorsal hand defects exposing the extensor tendons. In group I; defects were reconstructed using free muscle flaps (eight rectus abdominis and two latissimus dorsi) followed by skin grafting. In group II; defects were reconstructed by fascio-cutaneous flaps (eight reversed flow posterior interosseous artery and two reversed radial forearm flaps). All flaps healed well however one posterior interosseous artery flap suffered congestion and partial loss, one reversed radial forearm flap suffered disruption that was treated by secondary sutures and one skin graft over a free rectus flap that suffered partial loss.

Active and passive ranges of motion (ROM) for the metacarpo- phalangeal joint (MPJ) movement for both groups were measured. Concerning group I, the mean for passive ROM was $111.4 \pm 8.14$ degrees, the mean for active ROM was 75.4 434.11 degrees (Table 1). Concerning group II, the mean for passive ROM was 111.16 \pm 7.91 degrees, the mean for active ROM was $89.3 \pm 31.7$ degrees (Table 2). Passive and active ROM at the MPJ were meas- ured and the difference was calculated in the two groups and shown in Tables $(1,2)$.

Tenolysis was needed in four cases; two belonged to each group I, II. On comparing the values for active ROM for all patients in the two groups I and II, we found very weak inverse linear relationship by Pearson's correlation coefficient (Pearson's $r)=-0.135$ and also we found that probability of Null hypothesis ( $p$-value) $=0.687$; that means that, there is no significant difference in active ROM between the two groups at four months postoperatively.

\section{DISCUSSION}

Fascial and fascio-cutaneous flaps provide thin and mobile coverage with reliable vascular supply, potential for sensibility, and most importantly a gliding surface to facilitate tendon and joint mobility [19]. In large hand and forearm defects, fascial and fascio-cutaneous flaps may show some limitations especially in females, due to large donor sites. In case of large defects, muscle flap offers large surface area for coverage, with less cosmetic morbidity, as rectus and latissimus free muscle flaps.

In reconstruction of dorsal hand defects, it is very helpful to use muscle free flaps, but concerns may arise about the gliding surface of the tendons underneath the muscle flap. This study was intended to compare the gliding surface offered to the extensor tendons underneath fascio-cutaneous and muscle flaps on the dorsum of the hand. The study compared twenty patients with soft tissue defects over the dorsum of the hand with or without extension to the wrist and distal forearm.

In group I, we used free muscle flaps (eight rectus, two latissimus). All flaps were anastomosed to the radial artery and the cephalic vein except one flap, venous anastomoses was done to the basilic vein. The donor sites of the flaps were closed and no complications concerning the donor site occurred. All flaps healed well and the split thickness skin grafts were applied within range of 6-11 days. One graft suffered partial loss, while all other skin grafts healed uneventfully (Figs. 1,2). In group II fascio-cutaneous flaps used were the reversed flow posterior interosseous artery flap (8 cases) and the reversed radial forearm flap (2 cases), (Fig. 3). All flaps passed uneventful, except for partial loss in one posterior interosseous flap; that healed by secondary intension and one reversed radial forearm flap suffered from disruption and underwent secondary suturing. 


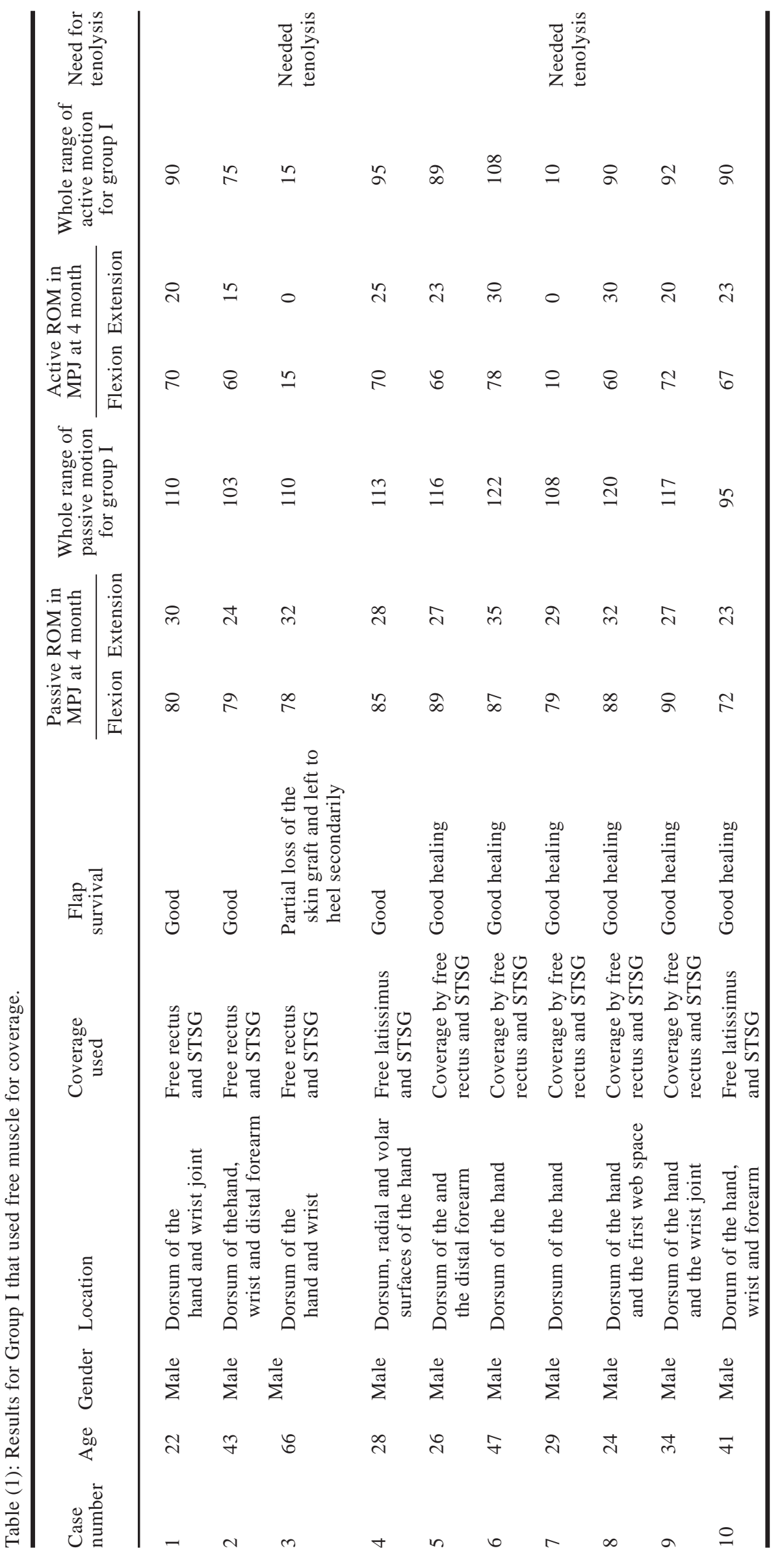




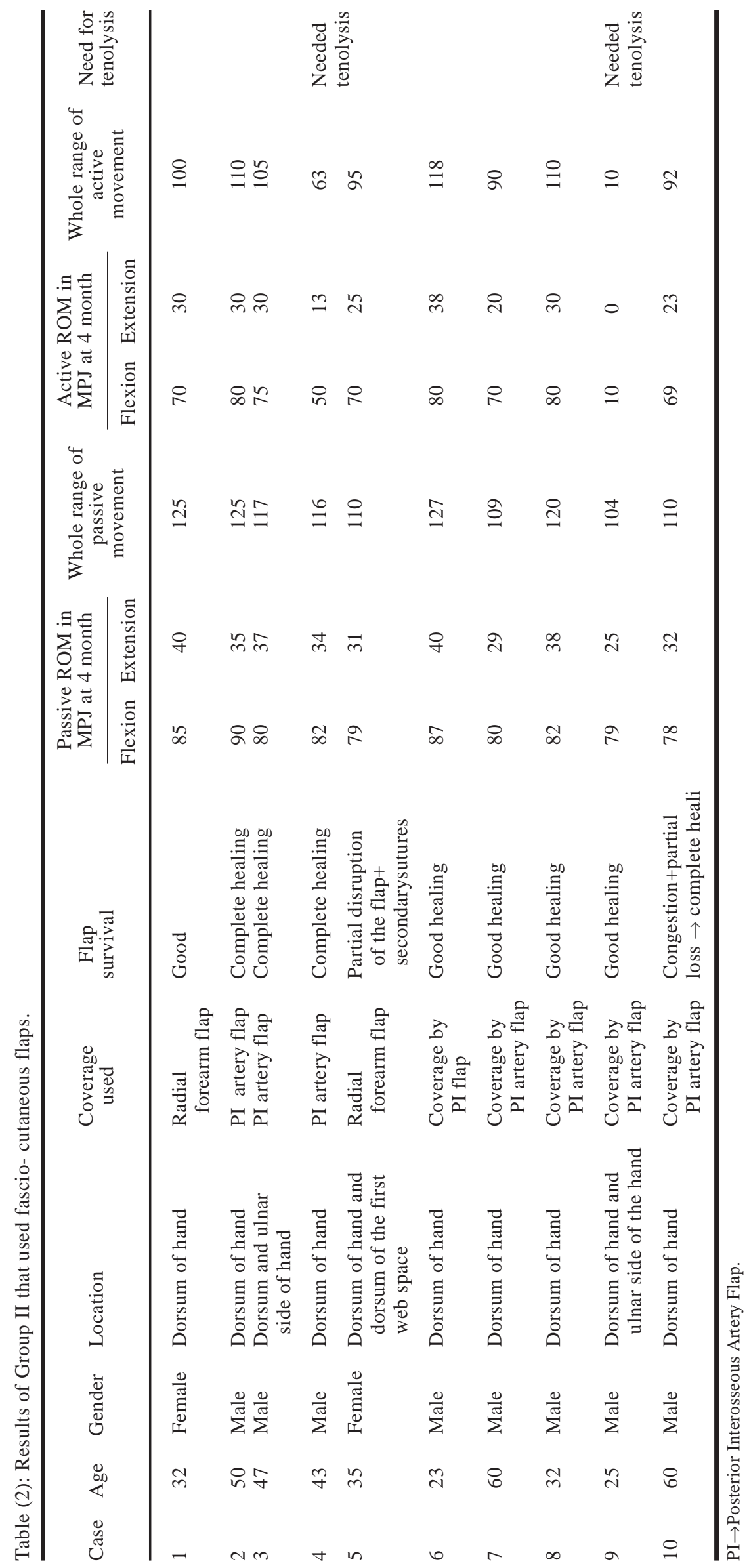



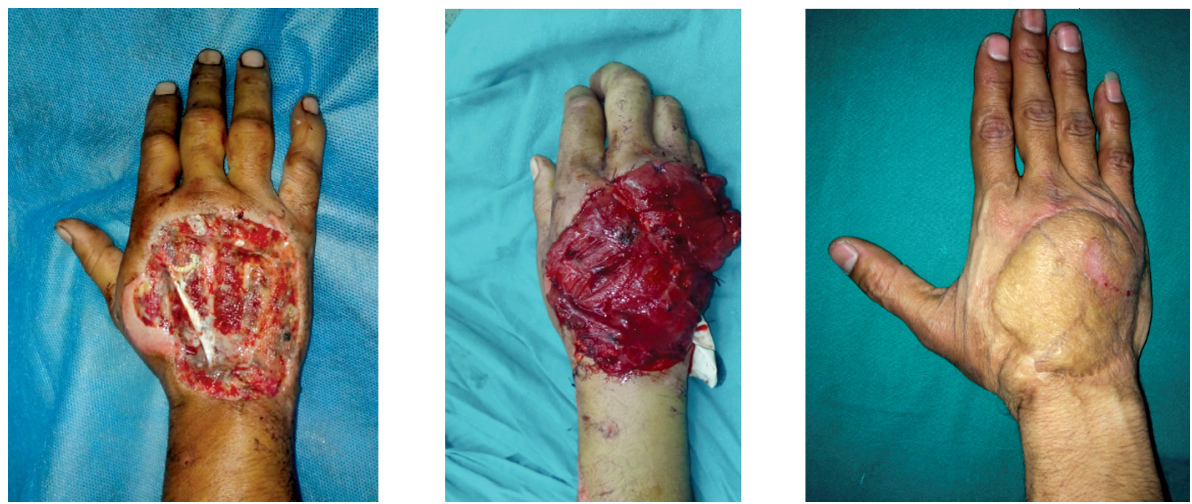

Fig. (1): 47 years old male patient; the photo to the left shows post traumatic avulsion hand defect. Middle photo shows free rectus flap inset. The photo to the right shows the flap after healing 42 days postoperatively.
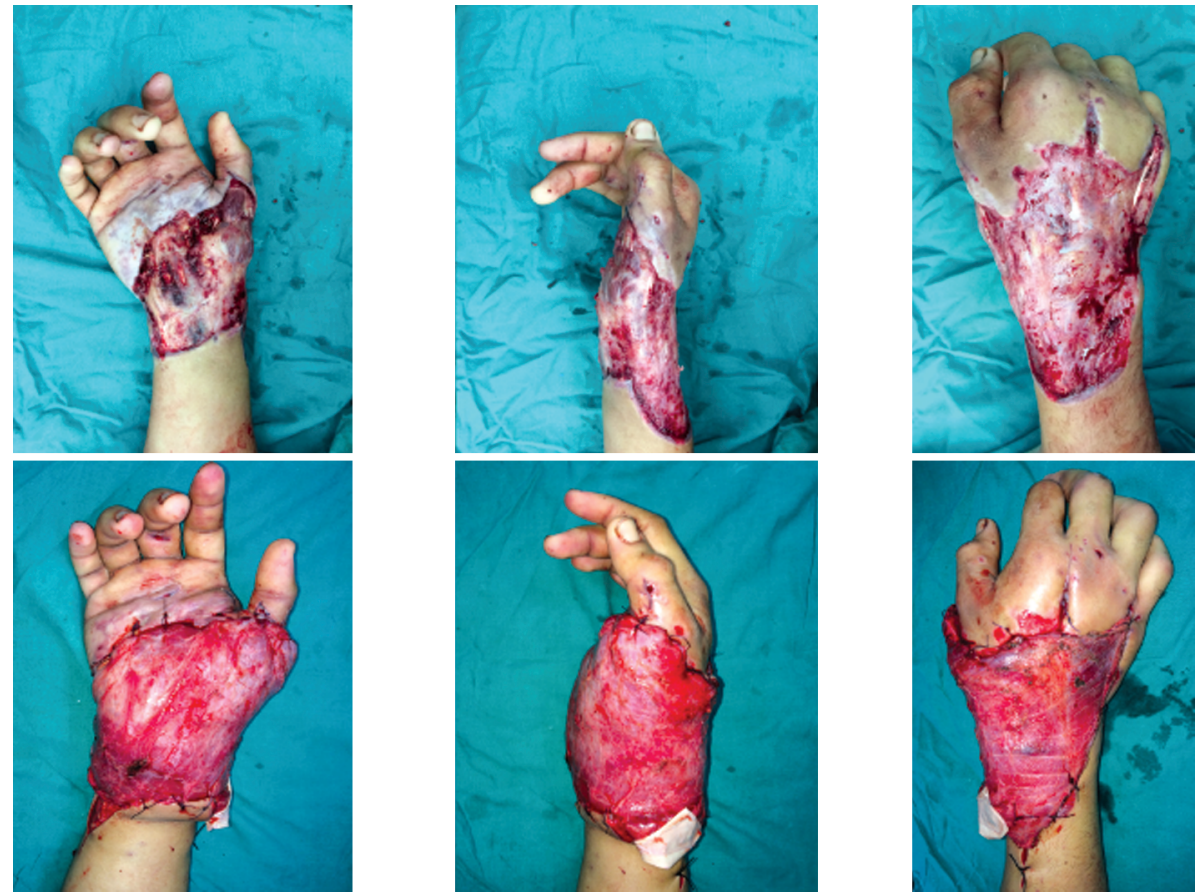

Fig. (2): 28 years old male patient; the upper photos shows the hand defect that runs from the dorsum of the hand, then along the radial side and the volar side of the hand. The lower photos show after free latissimus flap inset.
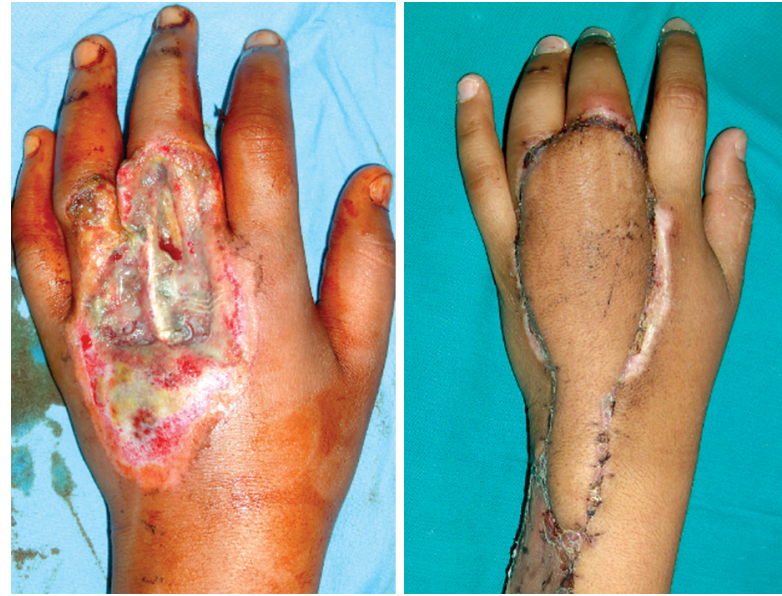

Fig. (3): 23 years old male patient; the left photo shows dorsal hand defect. The right photo shows reversed flow posterior interosseous artery flap inset.
Postoperative rehabilitation was a crucial step as it helped the hand regain its functions early and prevent adhesions [16,17]. Early postoperative physiotherapy was done following immobilization for the first 3-5days after flap inset that was required to enable flap healing [14]. Following immobilization, passive range of motion for all hand joints was applied [15]. Two weeks later on, scar stretching with active range of motion guided by pain was done [16]. Once healed, massage began gradually to soften and mobilize tissues. Rehabilitation coupled with ultrasound massaging improved patients joints and prevented forthcoming deformities [17]. Later on return to work, also graded rubber exercise bands and springs increased muscle strength [18]. 
In our study we have chosen the MPJ range of motion based on the fact that the amplitude of tendon excursion along the dorsum of the hand is the highest compared to the fingers that shows very low tendon excursion due to its intricate and delicate anatomy. Tendon excursion is the distance travelled by the tendon when the muscle is in complete action and complete relaxation. The amplitude of tendon excursion for the finger extensors is $5 \mathrm{~cm}$, most of it, occurs in the forearm, wrist and dorsum of the hand [20]; therefore the measurement of the flexion and extension of the MPJ reflects the tendon gliding over the dorsum of the hand. The normal range of motion of the MPJ is ninety degrees in flexion and forty five degrees in extension in average normal individuals. We measured the active ROM at the MPJ after four months of flap inset to measure the tendon gliding underneath the flaps, also we examined the passive ROM and calculated the difference (extensor lag) to define the need for tenolysis. In four cases; two belonging to each group, tenolysis was needed. The results of our study showed that there was no statistical significance (Pearson $r=-0.13, p$-value $=0.68$ ) between the active range of motion in the MPJ in both groups, therefore the use of muscle free flaps as a durable coverage for the dorsum of the hand didn't decline the extensor tendons gliding.

\section{Conclusion:}

Both the fasciocutaneous and muscle flaps are reliable as durable coverage for dorsal hand defects with no significant difference in underneath extensor tendon gliding.

\section{REFERENCES}

1- Carty M., Taghinia A. and Upton J., M.D.: Fascial Flap Reconstruction of the Hand: A Single Surgeon's 30-Year Experience. Plast. Reconstr. Surg., 125: 953, 2010.

2- Song R., Song Y., Yu Y., et al.: The upper arm free flap. Clin. Plast. Surg. 9: 27-35, 1982.

3- Reid C. and Moss L.: One-stage flap repair with vascularized tendon grafts in dorsal hand injury using the "Chinese" forearm flap. Br. J. Plast. Surg., 36: 473-479, 1983.

4- Upton J., Rogers C., Durham-Smith G., et al.: Clinical applications of the free temporo-parietal flaps in hand reconstruction. J. Hand. Surg. (Am.), 11: 475-483, 1986.
5- Becker C. and Gilbert A.: The ulnar flap. Handchir Mikrochir Plast. Chir., 20: 180-183, 1988.

6- Zancolli E.A. and Angrigiani C.: Posterior interosseous island forearm flap. J. Hand. Surg. (Br.), 13: 130-135, 1988.

7- Colen L., Pessa J., Potparic Z., et al.: Reconstruction of the extremity with the dorsal thoracic fascia free flap. Plast. Reconstr. Surg., 101: 738-744, 1998.

8- Bailey B. and Godfrey A.: Latissimus dorsi muscle free flaps. Br. J. Plast. Surg., 35: 47, 1982.

9- Jones N. and Lister G.: Free skin and composite flaps. In D. P. Green, R. N. Hotchkiss, and W. C. Pederson (Eds.), Operative Hand Surgery, $4^{\text {th }}$ Ed. Philadelphia: Churchill Livingstone, 1999.

10- Horch R. and Stark G.: The rectus abdominis free flap as an emergency procedure in extensive upper extremity soft-tissue defects. Plast. Reconstr. Surg., 103: 1421, 1999.

11- Rao V. and Baertsch A.: Microvascular reconstruction of the upper extremity with the rectus abdominis muscle. Microsurgery, 15: 746, 1994.

12- Parrett B., Bou-Merhi J., Buntic R., et al: Refining Outcomes in Dorsal Hand Coverage: Consideration of Aesthetics and Donor-Site Morbidity. Plast. Reconstr. Surg., 126: 1630,2010

13- Cooper C.: Fundamentals of Hand Therapy: Clinical Reasoning and Treatment Guidelines for Common Diagnoses of the Upper Extremity. Mosby Elsevier: St. Louis, Missouri, 2007.

14- Boscheinen-Morrin J. and Connolly W.: The Hand: Fundamentals of Therapy, $3^{\text {rd }}$ Ed, Butterworth-Heinemann: Oxford, 2001.

15- Glassey N.: Physiotherapy for burns and plastic reconstruction of the hand, United Kingdom: John Wiley \& Sons Ltd, 2004.

16- British Burns Association (BBA): National burn care Revieww, BBA: London, 2006.

17- Mohan G., Rajalaxmi V., Senthil V., et al.: Effectiveness of physiotherapy in skin grafting, International Journal of Physiotherapy \& Occupational Therapy, 2 (2): 33-38, 2016.

18- Herndon D.: Total burn care, $2^{\text {nd }}$ edn. WB saunders: London, 2002.

19- Matthew J. Carty, M.D., Amir Taghinia, M.D. and Joseph Upton, M.D.: Fascial Flap Reconstruction of the Hand: A Single Surgeon's 30-Year Experience. Plast. Reconstr. Surg., 125: 953, 2010.

20- Boyes J.H.: Bunnell's Surgery of the Hand, $5^{\text {th }}$ ed, Philadelphia, J.B. Lippincott, 1970. 